\title{
Innovationstätigkeit imMittelstand: FuE nicht zwingend erforderlich
}

\author{
Siegrun Brink ${ }^{1}$. Sebastian Nielen ${ }^{1}$
}

Received: 10 March 2021 / Accepted: 17 May 2021 / Published online: 8 June 2021

(c) The Author(s) 2021

\section{Zusammenfassung}

Die vorliegende Studie widmet sich der Innovationstätigkeit des Mittelstandes. Im Fokus stehen dabei nicht-forschende, mittelständischen Innovatoren. Diese meist kleinen Unternehmen sind vorrangig im Dienstleistungsbereich zu finden. Ziel der quantitativen Studie war es zu ermitteln, welche alternativen Strategien nicht-forschende Innovatoren umsetzen, um Innovationen zu generieren und die Frage zu beantworten, inwiefern diese eigene Forschung und Entwicklung ersetzen können. Die Ergebnisse unserer Untersuchung von 331 mittelständischen Unternehmen zeigen, dass der eigenen Forschungsund Entwicklungstätigkeit für die Erstellung von Produktinnovationen eine entscheidende Rolle zukommt. Dabei nimmt die Relevanz eigener Forschungs- und Entwicklungstätigkeit mit dem Neuigkeitsgrad der Produktinnovation zu. Vor allem radikale Produktinnovationen bedingen eigene Forschungs- und Entwicklungstätigkeit. Prozess- und nicht-technologische Innovationen sind hingegen auch ohne eigene Forschung und Entwicklung realisierbar. Als erfolgreiche Maßnahmen zum Generieren von Innovationen abseits der eigenen Forschung und Entwicklung erweisen sich die Aus- und Weiterbildung der eigenen Belegschaft, sowie Maßnahmen zur Digitalisierung und die allgemeine Investitionstätigkeit.

Schlagwörter Innovation $\cdot$ Neuartigkeit $\cdot \mathrm{FuE} \cdot$ nicht-forschende Unternehmen $\cdot$ Mittelstand (KMU)

JEL Classification $\mathrm{O} 31 \cdot \mathrm{O} 32$

\section{Einleitung}

Innovationen gelten seit jeher als einer der bedeutendsten Treiber von Volkswirtschaften. Die Entwicklung neuer Produkte, Dienstleistungen und Geschäftsprozesse - wie Innovationen gemeinhin nach Schumpeter (1961) verstanden werden - stehen für Wachstum, Fortschritt und eine Weiterentwicklung der Gesellschaft und sichern letztendlich die (internationale) Wettbewerbsfähigkeit von Unternehmen. Dabei entstehen Innovationen nicht nur durch technologische und gesellschaftliche Veränderungen, vielmehr ermöglichen sie auch den Umgang mit derartigen Veränderungen. Folglich erfährt das Thema Innovation auch in der wissenschaftlichen Diskussion seit langem große Aufmerksamkeit und es existiert eine Vielzahl wissenschaftlicher Arbeiten,

Sebastian Nielen

nielen@ifm-bonn.org

Siegrun Brink

brink@ifm-bonn.org

1 Institut Für Mittelstandsforschung (IfM) Bonn, Bonn, Germany die sich mit der Entstehung von Innovationen auseinandersetzen (Tidd et al. 2018; Pavitt 2003; Legler et al. 2005; Rammer et al. 2017).

Seit 2008 ist der Anteil der Unternehmen, die Produktoder Prozessinnovationen eingeführt haben, rückläufig, obwohl nicht nur die finanziellen Mittel, die Unternehmen in Innovationsvorhaben investiert haben, sondern auch die Erfolge, die Unternehmen mit Innovationen erzielt haben, gestiegen sind (Rammer et al. 2017). Als Schlüssel zur Hervorbringung und Durchsetzung von Innovationen werden insbesondere unternehmenseigene Forschung und Entwicklung (FuE) gesehen. Auf diesem Gebiet gelten Großunternehmen aufgrund ihrer höheren Sach- und Humankapitalausstattung als besser geeignet, um neues Wissen zu generieren, daraus Innovationen zu schaffen und diese in am Markt verwertbare Produkte und Dienstleistungen umzusetzen. Die Innovationsberichte der letzten Jahre vermitteln den Eindruck, dass insbesondere mittelständische Unternehmen zu wenig für Forschung ausgeben und damit im Wettbewerb zurückzufallen drohen. Demnach sei nicht nur die Innovationsdynamik im Mittelstand rückläufig (DIHK 2017), sondern auch die Innovationsintensität von KMU gehe zurück (EFI 2016). 
Ausschlaggebend für die Wettbewerbsfähigkeit eines Unternehmens ist jedoch nicht der Innovationsinput, sondern vielmehr der Innovationserfolg: Die Fähigkeit eines Unternehmens, ein neuartiges Produkt zu schaffen und am Markt durchzusetzen oder die eigenen Fertigungsprozesse zu erneuern und hierdurch Produktivitäts- und Kostenvorteile gegenüber Wettbewerbern zu erzielen, ist mitentscheidend, ob es gelingt, die eigene Marktposition national wie international zu sichern und weiter auszubauen (Maaß und Führmann 2012). Dabei kommt es nicht darauf an, wie Innovationen im Unternehmen entstehen bzw. entwickelt werden. Obwohl unternehmerischen FuE-Aktivitäten einen wesentlichen Beitrag zur Entwicklung von Innovationen leisten, können Innovationen letztlich in allen Bereichen eines Unternehmens entstehen. So beispielswiese auch bei der Bewältigung von Alltagsaufgaben und im Rahmen von Netzwerken oder Kooperationsbeziehungen mit anderen Unternehmen (Faix et al. 2014; Rammer et al. 2010; HirschKreinsen 2004).

Doch welche (alternativen) Strategien kommen abseits von FuE in nicht-forschenden mittelständischen Unternehmen zum Einsatz, um Innovationen hervorzubringen? Hier setzt unser Beitrag an: Auf der Basis einer empirischen Untersuchung gehen wir dieser Frage nach. Um über die Innovationsfähigkeit von nicht-forschenden mittelständischen Unternehmen diskutieren zu können, müssen wir zunächst fragen, was ,nicht-forschend “ bedeutet (Kapitel 2.1) und erörtern, welche Besonderheiten mittelständische Unternehmen aufweisen und welche Bedeutung Innovationen für diese Unternehmen haben (Kapitel 2.2). Anschließend stellen wir in Kapitel 3 unsere empirische Erhebung vor, um die daraus gewonnenen Ergebnisse in Kapitel 4 zu diskutieren. Der Beitrag schließt mit einem Fazit und einem Ausblick auf weitere Forschungsbedarfe (Kapitel 5).

\section{Konzeptioneller Rahmen}

\subsection{FuE-basierte vs. nicht-forschungsbasierte Innovationen}

Als FuE-Aktivitäten werden eine gezielte Ausschöpfung, Schaffung und (Re-)Kombination von unternehmensinternen Wissensbeständen bezeichnet. Die Kernaufgabe besteht in einer systematischen Suche nach neuen und besseren Möglichkeiten am Markt zu bestehen und die Wettbewerbsfähigkeit zu steigern (Erdmann et al. 2012). Bei der Entwicklung (technischer) Neuerungen im Rahmen von unternehmerischen FuE-Aktivitäten wird vor allem auf wissenschaftliche und formalisierte Wissensbestände im Unternehmen zurückgegriffen. Im klassisch verlaufenden Innovationsprozess werden auf der Basis von Inventionen Prototypen entwickelt und getestet. Diese werden dann bis zur Serienreife weiterentwickelt und letztendlich in marktfähige Produkte oder einsetzbare Herstellungsprozesse überführt (Pavitt 2003; Rammer et al. 2017).

Kennzeichnend für FuE sind insbesondere hohe Einstiegskosten für technische, räumliche und personelle Ausstattung, die darüber hinaus nicht beliebig skalierbar sind. Entsprechend müssen FuE-basierte Innovationsvorhaben eine kritische Mindestgröße erreichen, damit sie sich für ein Unternehmen betriebswirtschaftlich lohnen. Bei den dabei entstehenden Kosten handelt es sich überwiegend um Fixkosten, die unabhängig von der tatsächlichen, erfolgreichen Entwicklung von Innovationen anfallen. Einnahmen aus Innovationserfolgen werden hingegen erst zeitlich versetzt erwirtschaftet. FuE-Aktivitäten sind für die Unternehmen entsprechend mit hohen Risiken und Unsicherheiten verbunden (Cohen und Klepper 1996; Czarnitzki und Kraft 2006).

Von diesen, auf der Basis von FuE gezielt entwickelt und als FuE-basiert zu bezeichnenden Innovationen, sind nicht-forschungsbasierte Innovationen zu unterscheiden: Nicht-forschungsbasierte Innovationen entstehen folglich ohne explizite Forschungsorientierung und -budgets im alltäglichen Arbeitsprozess. Dabei wird auf Erfahrungen, Fähigkeiten und Fertigkeiten der Mitarbeiter aus dem Anwendungskontext zurückgegriffen, indem beispielsweise Grundbausteine bestehender Produkte so kombiniert werden, dass neuartige Anwendungen entstehen (sog. learning by doing, using and interacting) (Hirsch-Kreinsen 2004; Rammer et al. 2010; Lundvall und Johnson 2017). Wissensträger sind in diesem Zusammenhang jedoch nicht nur Produktionsmitarbeiter oder das Management des Unternehmens selbst, sondern auch verschiedenste andere Personen und -gruppen, vom Lieferanten, Kooperationspartner bis hin zum Endkunden (Hirsch-Kreinsen 2004).

Auf welche Weise ein Unternehmen innoviert - FuEbasiert oder nicht-forschend - hängt nicht zuletzt von den zur Verfügung stehenden unternehmerischen Ressourcen, aber auch von den organisatorischen und unternehmerischen Rahmenbedingungen eines Unternehmens ab. Insbesondere zwischen mittelständischen und großen Unternehmen zeigen sich Unterschiede in Innovationsmodellen und -aktivitäten, die wir nachfolgend in den Mittelpunkt rücken.

\subsection{Bedeutung von Innovationen für mittelständische Unternehmen}

Im Jahr 2017 haben insgesamt 36,0\% aller deutschen Unternehmen Produkt- oder Prozessinnovationen eingeführt (Rammer et al. 2019). Während dies mit 67,7\% auf eine deutliche Mehrheit der Großunternehmen zutraf, zählten unter den kleinen und mittleren Unternehmen (KMU) lediglich $35,0 \%$ zu den Innovatoren. Mit zehn bis $15 \%$ 
entfällt lediglich ein geringer Anteil der deutschen FuEbzw. Innovationsausgaben auf KMU (Rammer et al. 2017, S. 199). Diese Zahlen sprechen zunächst eine eindeutige Sprache: Nicht nur der Anteil der Innovatoren unter den KMU ist deutlich geringer als bei Großunternehmen. Im Vergleich zu Großunternehmen fließen in KMU auch weniger finanzielle Mittel in die Entwicklung von Innovationen (Rammer et al. 2020).

Allerdings weisen bisherige Studien, die sich mit der Messung der Innovationstätigkeit auseinandersetzen, wesentliche Limitationen auf: Zum einen wird zur Messung der unternehmerischen Innovationstätigkeit häufig der FuEAufwand eines Unternehmens als Messgröße herangezogen (Maaß und Führmann 2012). Die Fokussierung dieses InputIndikators vernachlässigt jedoch solche Innovationen, denen keine formalen FuE-Aktivitäten zugrunde liegen und die abseits von FuE entstehen (Handke 2005; Hirsch-Kreinsen 2004). Da für die Wettbewerbsfähigkeit eines Unternehmens jedoch nicht der Innovationsinput, sondern vielmehr der Innovationserfolg entscheidend ist, scheint diese Herangehensweise weniger geeignet, um das tatsächliche Innovationsgeschehen abzubilden. Es führt tendenziell eher zu einer Untererfassung der unternehmerischen Innovationstätigkeit, die insbesondere zu Lasten des Mittelstands geht (Maaß und Führmann 2012; Brink et al. 2014).

Zum anderen wird zur Abgrenzung mittelständischer Unternehmen vorwiegend die Unternehmensgröße genutzt, während die Einheit von Eigentum und Leitung als qualitatives Bestimmungsmerkmal mittelständischer Unternehmen unberücksichtigt bleibt. In mittelständischen Unternehmen halten per definitionem ,... bis zu zwei natürliche Personen oder ihre Familienangehörigen (direkt oder indirekt) mindestens 50\% der Anteile eines Unternehmens. [D]iese natürlichen Personen gehören der Geschäftsführung an " (IfM Bonn 2020). Es ist genau dieser Mittelstand, der im internationalen Vergleich - beispielsweise mit seinen Hidden Champions - durchaus erfolgreich und konkurrenzfähig ist. Obwohl die Schnittmenge von mittelständischen Unternehmen sowie KMU groß ist, zeigen Maaß und MayStrobl (2016, S. 16), dass sich eigenständige und gruppenzugehörige KMU erheblich in ihrem Innovationsverhalten unterscheiden. So haben mittelständische Unternehmen, in welchen Eigentum und Leitung in einer Hand liegen, im Vergleich zu gruppenzugehörigen KMU nicht die Möglichkeit auf Ressourcen (Personal, finanziellen Mittel) oder Strukturen (FuE-Abteilung) des Mutterkonzern zurückzugreifen. Einerseits führt diese geringere Ausstattung mit Ressourcen aufgrund der strukturellen Merkmale von unternehmenseigenen FuE-Aktivitäten zu einer geringeren FuE-Neigung bei (eigenständigen) kleinen und mittleren Unternehmen als bei Großunternehmen (Shefer und Frenkel 2005). Für mittelständische Unternehmen kann das Scheitern bereits eines einzelnen FuE-basierten Innovationsprojektes die
Existenz des gesamten Unternehmens gefährden (Rammer et al. 2017).

Andererseits weisen mittelständische Unternehmen meist einen geringeren Formalisierungsgrad und flachere Hierarchien auf als Großunternehmen, die schnellere und flexiblere Entscheidungsprozesse ermöglichen. Dies kann insbesondere bei der Entwicklung von Innovationen vorteilhaft sein. Entsprechend steht der Theorie des "NichtKönnens" aufgrund einer ressourcenbezogenen Mangelsituation von Unternehmen die Theorie der bewussten, strategischen Entscheidung (Ressource- bzw. Knowledgebased-view) gegenüber. Nach diesem Verständnis erfolgt die Aufnahme eigener FuE-Anstrengungen nur dann, wenn die benötigten Wissensressourcen nicht in ausreichendem Maße im Unternehmen verfügbar sind und auch nicht aus dem Unternehmensumfeld, bspw. über Kooperationen oder Interaktionen mit externen Partnern, erschlossen werden können (Rammer et al. 2017). Unklar bleibt dabei bisher, welche alternativen Strategien mittelständische Unternehmen einsetzen, um diese Wissensbestände im Unternehmen zu generieren. Hier setzt der vorliegende Beitrag an und beantwortet auf der Grundlage eines explorativen Forschungsansatzes die Frage, auf welchem Weg mittelständische Unternehmen ohne formale FuE-Aktivitäten Innovationen entwickeln. Im Mittelpunkt stehen dabei die Fragen, welche Arten von Innovationen diese Unternehmen hervorbringen, durch welche Merkmale die Unternehmen gekennzeichnet sind und welche alternativen Pfade sie wählen, um konkurrenzfähig zu bleiben.

\section{Empirische Erhebung}

\subsection{Daten und Variablen}

Die vorliegende Studie verwendet Daten, die aus einer eigenen Unternehmensbefragung stammen. Im Zuge dessen wurden aus dem Bestand der Datenbanken der Creditreform e.V. Unternehmen geschichtet nach Branche und Größenklasse gezogen. Die Gesamtstichprobe setzt sich aus zwei Teilstichproben zusammen. Teilstichprobe eins wurde vollständig zufällig gezogen. Bei der zweiten Teilstichprobe handelt es sich um schnell wachsende Unternehmen. Das heißt, die gezogenen Unternehmen mussten im Zeitraum von 2010 bis 2013 ein Wachstum von mindestens 72,8\% aufweisen. Dieses Kriterium basiert auf Studien der OECD (Eurostat und OECD 2007; OECD 2010): Diese stufen Unternehmen als schnell wachsend ein, wenn in drei aufeinander folgenden Jahren ein Wachstum von mindestens 20\% erreicht wird. Über drei Jahre hinweg ergibt sich damit ein Wachstum von insgesamt mindestens $72,8 \%$ (Eurostat und 
OECD 2007). Unter allen Unternehmen, die diese Bedingung erfüllten, wurde dann auch zufällig gezogen. ${ }^{1}$

Die Befragung fand im Zeitraum von Oktober 2015 bis Januar 2016 online statt. Insgesamt haben an der Befragung 693 Unternehmen teilgenommen. Allen Unternehmen haben, unabhängig von der Zugehörigkeit zu einer der Teilstichproben, einen einheitlichen Fragebogen ausgefüllt. Das entspricht einer Beteiligungsquote von 4,8\%. Im Rahmen der Datenaufbereitung wurden 224 Unternehmen wegen teilweise fehlender Angaben zum Umsatz oder zur Anzahl der Beschäftigten ausgeschlossen. Weitere 138 Unternehmen mussten ausgeschlossen werden, da diese entweder den Kriterien eines mittelständischen Unternehmens nicht entsprechen oder in mindestens einer der verwendeten Variablen einen fehlenden Wert aufweisen. Somit verblieben für die finale Stichprobe 331 mittelständische Unternehmen mit vollständig ausgefüllten Fragebögen. In der Datenbasis ist jedes Unternehmen nur einmal enthalten. Die Stichprobe unserer Studie verteilt sich auf die beiden Gruppen wie folgt: 170 Unternehmen gehören zur Gruppe der schnell wachsenden Unternehmen und die verbleibenden 161 zur anderen Gruppe.

Um zwischen forschenden und nicht-forschenden mittelständischen Unternehmen zu unterscheiden wird auf eine Dummyvariable zurückgegriffen. Im Fragebogen wurden die Unternehmen gefragt, ob sie in den vergangenen fünf Jahren FuE-Maßnahmen ergriffen haben oder nicht. Die darauf basierende Dummyvariable nimmt den Wert eins an, wenn das betreffende Unternehmen FuE-Maßnahmen ergriffen hat, ansonsten ist sie entsprechend null. Darüber hinaus enthält der Fragebogen Informationen bezüglich der Innovationstätigkeit der befragten Unternehmen. Auch diese Fragen beziehen sich auf den Zeithorizont der vergangenen fünf Jahre. Hinsichtlich des Innovationsoutputs wird zwischen den folgenden Innovationsarten unterschieden:

- Implementierung mindestens einer Innovation, egal welcher Art (Innovation)

- Produkt/Dienstleistung ist neu aus Sicht des Marktes (Marktneuheit)

- Produkt/Dienstleistung ist neu aus Sicht des Unternehmens (Unternehmensneuheit)

- Weiterentwicklung bestehender Produkte/Dienstleistungen (Weiterentwicklung)

- Prozessinnovation

- Nicht-technologische Innovationen, wie bspw. Marketing- oder Organisationsinnovationen (Trantow et al. 2011; OECD/Eurostat 2003)

\footnotetext{
${ }^{1}$ Diese Unterteilung der Gesamtstichprobe erfolgte, da der Datensatz im Zuge einer Studie zu schnell wachsenden Unternehmen erstellt wurde.
}

Die Innovationsvariablen sind jeweils binär und nehmen den Wert eins an, wenn das Unternehmen die entsprechende Innovationsart erfolgreich implementiert hat. Im Zuge der Untersuchung alternativer Strategien zur Implementierung von Innovationen werden die folgenden strategischen Maßnahmen analysiert:

- Aus- und Weiterbildung der Belegschaft (Aus-/Weiterbildung)

- Digitalisierung von Produktion und Vertrieb (Digitalisierung)

- Investitionen in neue Anlagen oder Technologien (Investition)

- Kooperation mit Partnern aus Wirtschaft oder Wissenschaft (Kooperation)

- Inanspruchnahme externer Beratung jeglicher Art (Beratung).

Die jeweiligen Dummyvariablen nehmen den Wert eins an, wenn das betroffene Unternehmen die entsprechende strategische Maßnahme durchgeführt hat. Auch hier umfasst der jeweilige Zeithorizont die vergangenen fünf Jahre. Im Rahmen der Regressionsmodelle werden außerdem die folgenden Kontrollvariablen mitberücksichtigt: Anzahl der Mitarbeiter, Alter, Exporttätigkeit, Region und Branche. Darüber hinaus nehmen wir eine Dummyvariable bezüglich der wettbewerblichen Ausrichtung in die Regressionsmodelle mit auf. Diese nimmt den Wert eins an, wenn das betreffende Unternehmen sich ausschließlich über die Qualität der eigenen Produkte und Dienstleistungen am Markt positioniert und nicht über den Preis oder Preis und Qualität.

\subsection{Deskriptive Statistiken}

Tabelle 1 beinhaltet die Ergebnisse deskriptiver Auswertungen aller Innovationsvariablen, sowie alternativer strategischer Maßnahmen im Innovationsprozess und der Kontrollvariablen.

Annährend vier von fünf Unternehmen unserer Stichprobe gelang es mindestens eine Art von Innovationen erfolgreich umzusetzen. Erwartungsgemäß nimmt mit steigendem Neuheitsgrad der Innovation der Anteil der Unternehmen, die diese Art von Innovation hervorbringen konnte, ab. Prozessinnovationen wurden von etwas mehr als einem Drittel der Unternehmen in unserer Stichprobe umgesetzt. Etwas mehr als drei Viertel der Unternehmen bilden ihre eigenen Beschäftigten regelmäßig aus und weiter. Jeweils ungefähr 30\% führten Maßnahmen zur Digitalisierung durch, kooperierten mit Partnern aus Wissenschaft oder Wirtschaft oder nahmen externe Beratung in Anspruch. Im Durchschnitt beschäftigen die Unternehmen unserer Stichprobe ungefähr 136 Mitarbeiter und sind fast 33 Jahre alt. Ungefähr die Hälfte aller Unternehmen exportiert seine 
Tabelle 1 Deskriptive Ergebnisse: Innovationsarten und alternative Strategien

\begin{tabular}{|c|c|c|}
\hline Variable & Mittelwert & $\begin{array}{l}\text { Stand- } \\
\text { ardabwei- } \\
\text { chung }\end{array}$ \\
\hline Innovation & 0.792 & 0.407 \\
\hline Marktneuheit & 0.193 & 0.400 \\
\hline Unternehmensneuheit & 0.441 & 0.497 \\
\hline Weiterentwicklung & 0.625 & 0.485 \\
\hline Prozessinnovation & 0.347 & 0.477 \\
\hline Nicht-technologische Innovation & 0.145 & 0.353 \\
\hline Aus-/Weiterbildung & 0.764 & 0.425 \\
\hline Investition & 0.613 & 0.488 \\
\hline Digitalisierung & 0.311 & 0.464 \\
\hline Kooperation & 0.278 & 0.449 \\
\hline Beratung & 0.287 & 0.453 \\
\hline Qualität & 0.728 & 0.446 \\
\hline Mitarbeiter & 136,091 & 498,263 \\
\hline Alter & 32,837 & 34,012 \\
\hline Export: Ja & 0.511 & 0.500 \\
\hline Region: Westdeutschland & 0.831 & 0.375 \\
\hline Produzierendes Gewerbe & 0.396 & 0.490 \\
\hline Distribution & 0.217 & 0.413 \\
\hline Unternehmensnahe Dienstleistungen & 0.290 & 0.454 \\
\hline Sonstige Dienstleistungen & 0.097 & 0.296 \\
\hline
\end{tabular}

Quelle: Eigene Berechnungen
4 Ergebnisse der empirischen Untersuchung

\subsection{Innovationsarten nicht-forschender, mittelständischer Unternehmen}

Ein Blick auf die Innovationsarten, die mit und ohne FuEAktivitäten von mittelständischen Unternehmen hervorgebracht werden, zeigt die Bedeutung von FuE als Treiber für den unternehmerischen Neuerungsprozess. Bei allen Innovationsarten ist der Anteil der Innovatoren mit FuE höher als der ohne FuE (vgl. Abbildung 1).

Innovationen im Mittelstand erstrecken sich überwiegend auf Produkt- und Prozessinnovationen. Bei der Implementierung solcher technologischer Innovationen werden aber durchaus Unterschiede in der Abhängigkeit von FuEAktivitäten sichtbar. Der nicht-forschende Mittelstand ist auf die Verbesserung bestehender Produkte oder Dienstleistungen fokussiert $(53,1 \%)$. Hierbei handelt es sich um inkrementelle Innovationen, die in der Regel als kontinuierliche Veränderung bestehender Produkte/Dienstleistungen innerhalb einer Entwicklungslinie zu verstehen sind (Freeman 1992). Ihnen stehen radikale, diskontinuierliche Innovationen in Form völlig neuartiger Produkte/Dienstleistungen gegenüber, die lediglich von $12 \%$ der innovierenden, mittelständischen Unternehmen ohne FuE hervorgebracht werden. Im Gegensatz zu inkrementellen Innovationen, die mit geringeren Veränderungen einhergehen, entste-

Abbildung 1 Innovationsarten innovierender, mittelständischer Unternehmen. Quelle: Eigene

Berechnungen

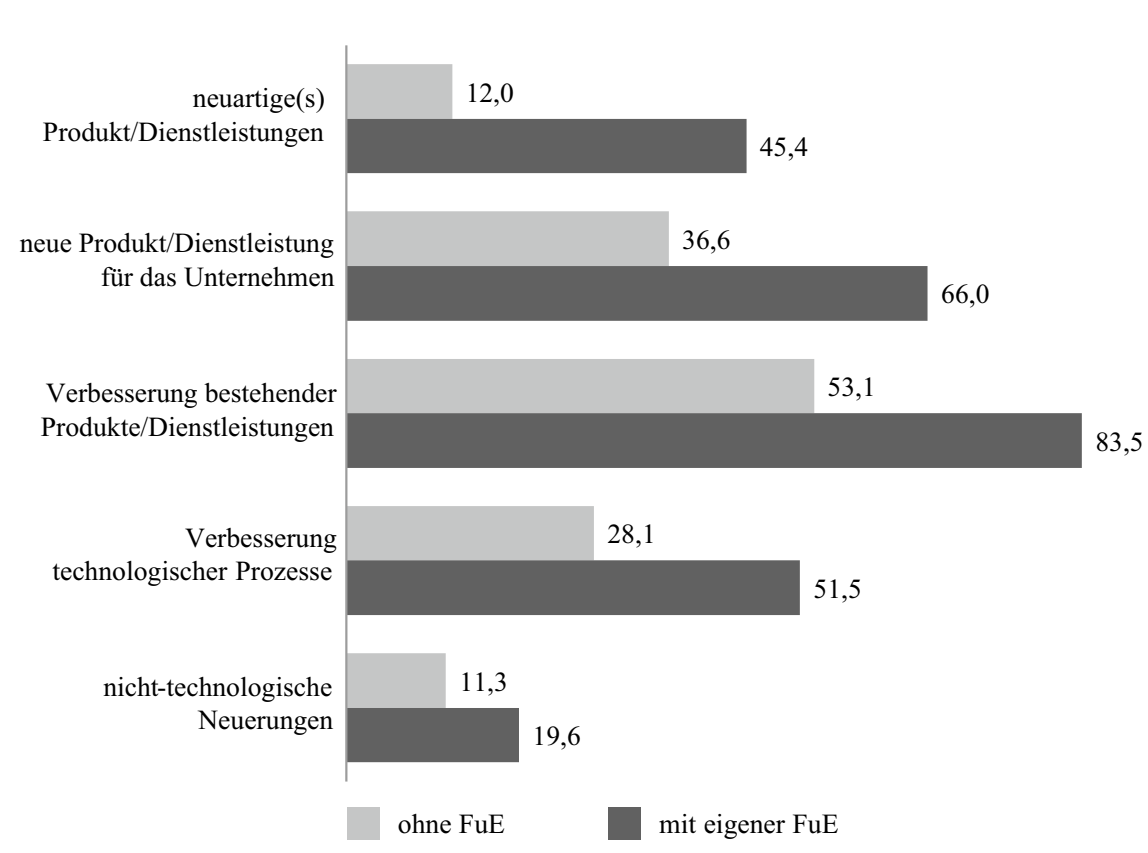

Waren oder Dienstleistungen auch in andere Länder. Eine deutliche Mehrheit der mittelständischen Unternehmen hat seinen Sitz in Westdeutschland. Die meisten Unternehmen sind im Produzierenden Gewerbe tätig. hen aus strategisch ausgerichteten FuE-Aktivitäten heraus häufig radikale Veränderungen (Freeman und Perez 1988). Dies gilt auch im Mittelstand - Marktneuheiten, denen in 


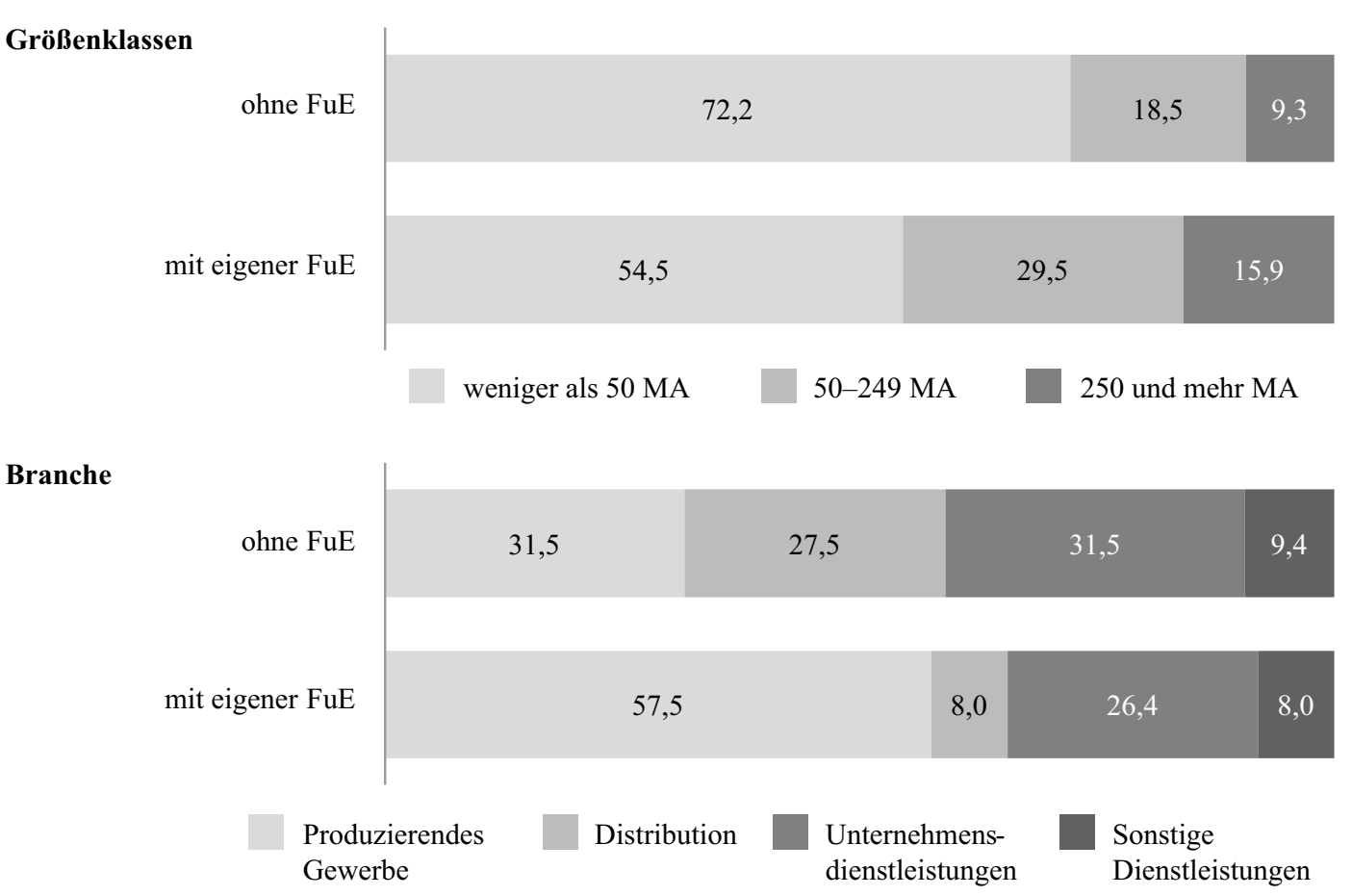

Produzierendes Gewerbe dienstleistungen

Abbildung 2 Merkmale innovativer, mittelständischer Unternehmen mit und ohne FuE im Vergleich. Quelle: Eigene Berechnungen

der Regel ein größeres ökonomisches Potential unterstellt wird, setzen in hohem Maße Aufwand in Forschung und Entwicklung voraus. Für die inkrementelle Weiterentwicklung von Produkten und Dienstleistungen sind formale FuEAktivitäten hingegen weniger bedeutsam. Mit der Neuartigkeit der Innovation steigt folglich die Notwendigkeit der Unternehmen, auf wissenschaftlich-technisches Wissen in Form von FuE zurückgreifen zu können, über das nichtforschende Unternehmen nicht verfügen.

Auch die Entwicklung von Prozessinnovationen wird häufig mit dem Vorhandensein technologischer Kompetenz in Form von unternehmensinternen FuE-Aktivitäten verknüpft (Tidd et al. 2018). Die vorliegenden Ergebnisse zeigen jedoch, dass Unternehmen in diesem Innovationsbereich durchaus auch nicht-FuE-basiert innovieren. Ein geringer Einfluss der FuE ist für nicht-technologische Innovationen zu konstatieren. Unabhängig von FuE-Aktivitäten werden die hierzu zählenden organisatorischen oder Marketinginnovationen aber vergleichsweise selten (von weniger als jedem fünften Unternehmen) durchgeführt.

Eine Möglichkeit, ohne FuE zu innovieren, stellen Interaktionen mit anderen Unternehmen und Kunden dar. Rund vier von zehn Unternehmen innovieren, indem sie Produkte/ Dienstleistungen, die bereits am Markt existieren, neu in ihr Produkt- oder Dienstleistungsangebot aufnehmen. Auf diese Weise profitieren die Unternehmen von der Diffusion und der Verfügbarkeit externer, neuer Wissensbestände, die in den adaptierten Produkten/Dienstleistungen enthalten sind. Zwar beinhaltet dies unter Umständen ein hohes Maß an technologischer Aufnahme- und Anschlussfähigkeit, die aber nicht notwendigerweise an interne FuE-Aktivitäten gekoppelt sein müssen (Bender und Laestadius 2005; Rammer et al. 2010). Zusammenfassend stellen wir fest, dass FuE umso bedeutsamer für die Entwicklung von Innovationen ist, je neuartiger und technologiegetriebener diese sind.

\subsection{Merkmale nicht-forschender, mittelständischer Innovatoren}

Es ist davon auszugehen, dass sich mittelständische Innovatoren ohne eigene FuE-Tätigkeit im Hinblick auf bestimmte Unternehmenscharakteristika von denen unterscheiden, die ihre Innovationen mit FuE generieren. Unterschiede lassen sich sowohl im Hinblick auf die Unternehmensgrößen als auch die Branchenstruktur nachweisen (vgl. Abbildung 2).

Mittelständische Innovatoren ohne eigene FuE sind tendenziell kleiner als mittelständische Innovatoren mit FuE. Hier bestätigt sich die Größenabhängigkeit von FuE-getriebenen Innovationen. Wir finden nicht-forschende, mittelständische Innovatoren im distributiven Sektor häufiger als im Verarbeitenden Gewerbe. Kaum Unterschiede bestehen hingegen bei den Unternehmensnahen Dienstleistungen. Insgesamt zeigen die Ergebnisse, dass vor allem für große Unternehmen, die im Verarbeitenden Gewerbe tätig sind, eigene Forschung und Entwicklung im Innovationsprozess eine entscheidende Rolle spielt. Unternehmen im Bereich 
Tabelle 2 Multivariate Ergebnisse: Strategien mittelständischer Unternehmen ohne eigene FuE

\begin{tabular}{|c|c|c|c|c|c|c|}
\hline & (1) & (2) & (3) & (4) & (5) & (6) \\
\hline Variablen & Innovation & Markt-neuheit & $\begin{array}{l}\text { Unternehmensneu- } \\
\text { heit }\end{array}$ & Weiterent-wicklung & Prozess-innovation & Nicht-technologisch \\
\hline Ausbildung & $0.092(0.062)$ & $0.086(0.055)$ & $-0.005(0.072)$ & $0.128 *(0.072)$ & $0.135 * *(0.067)$ & $0.152 * * *(0.056)$ \\
\hline Investitionen & $0.042(0.053)$ & $-0.000(0.038)$ & $0.112 *(0.061)$ & $0.031(0.064)$ & $0.175 * * *(0.055)$ & $0.028(0.043)$ \\
\hline Digitalisierung & $0.105 *(0.060)$ & $0.019(0.041)$ & $0.046(0.066)$ & $0.035(0.069)$ & $0.226 * * *(0.056)$ & $0.072 *(0.042)$ \\
\hline Kooperationen & $0.071(0.066)$ & $0.097 * *(0.039)$ & $-0.005(0.074)$ & $0.171 * *(0.074)$ & $0.024(0.062)$ & $-0.023(0.043)$ \\
\hline Beratung & $0.016(0.063)$ & $0.055(0.040)$ & $0.064(0.070)$ & $-0.026(0.073)$ & $0.003(0.062)$ & $0.070 *(0.040)$ \\
\hline Nur Qualität & $-0.011(0.059)$ & $0.049(0.045)$ & $-0.035(0.069)$ & $0.054(0.070)$ & $-0.045(0.060)$ & $0.011(0.046)$ \\
\hline $\log ($ Mitarbeiter $)$ & $0.060 * * *(0.021)$ & $0.018(0.014)$ & $0.025(0.021)$ & $0.033(0.022)$ & $0.009(0.018)$ & $0.021(0.014)$ \\
\hline $\log$ (Alter) & $-0.044(0.034)$ & $-0.045^{*}(0.026)$ & $0.021(0.040)$ & $-0.064(0.039)$ & $-0.053(0.039)$ & $0.022(0.025)$ \\
\hline Export: Ja & $0.113 * *(0.054)$ & $0.001(0.038)$ & $0.087(0.062)$ & $0.119 *(0.063)$ & $0.059(0.054)$ & $0.069 *(0.040)$ \\
\hline West & $0.114 *(0.068)$ & $0.001(0.048)$ & $0.080(0.080)$ & $0.055(0.082)$ & $0.064(0.069)$ & $0.022(0.053)$ \\
\hline Distribution & $0.006(0.066)$ & $0.171 * * *(0.059)$ & $0.100(0.082)$ & $-0.001(0.082)$ & $-0.234 * * *(0.065)$ & $0.064(0.051)$ \\
\hline $\begin{array}{l}\text { unt. Dienstleistun- } \\
\text { gen }\end{array}$ & $0.017(0.063)$ & $0.076^{*}(0.043)$ & $0.006(0.075)$ & $0.018(0.077)$ & $0.002(0.074)$ & $0.039(0.044)$ \\
\hline $\begin{array}{l}\text { sonst. Dienstleis- } \\
\text { tungen }\end{array}$ & $-0.161(0.107)$ & $-0.009(0.041)$ & $0.120(0.114)$ & $-0.059(0.113)$ & $-0.209 * *(0.087)$ & $0.099(0.075)$ \\
\hline Pseudo R-Quadrat & 0.11 & 0.02 & 0.05 & 0.06 & 0.16 & 0.17 \\
\hline Beobachtungen & 251 & 251 & 251 & 251 & 251 & 251 \\
\hline
\end{tabular}

Quelle: Eigene Berechnungen, Standardfehler in Klammern, Signifikanzniveaus: *** $\mathrm{p}<0.01, * * \mathrm{p}<0.05, * \mathrm{p}<0.1$

Distribution und Unternehmensnahe Dienstleistungen sind hingegen eher in der Lage, ohne eigene FuE Innovationen hervorzubringen. Ebenso verhält es sich bei Kleinst- und Kleinunternehmen. Die Hinweise von Maaß und May-Strobl (2016), in Bezug auf die Innovationsberichterstattung eine größen- und branchenbezogene Verbreiterung des Blickwinkels einzunehmen, finden hiermit eine weitere empirische Fundierung.

\subsection{Alternative Strategien nicht-forschender, mittelständischer Innovatoren}

Auf welche Art und Weise aber gelingt es mittelständischen Unternehmen ihre Innovationsvorhaben umzusetzen, wenn nicht mit Hilfe eigener FuE-Aktivitäten? Dieser Frage soll in der folgenden Analyse nachgegangen werden. Dabei wird untersucht, welche Maßnahmen sich wie auf die Innovationstätigkeit auswirken. Um dieser Frage nach zu gehen schätzen wir für jede Innovationsart ein separates ProbitModell, welches die Wahrscheinlichkeit der Implementierung der entsprechenden Innovationsart als abhängige Variablen enthält. Die folgende Tabelle zeigt die entsprechenden (durchschnittlichen) marginalen Effekte jedes einzelnen Models (Tabelle 2).

Strategische Maßnahmen, die am ehesten mit der Generierung von Innovationen einhergehen, sind Aus- und Weiterbildung sowie Digitalisierung. Der Einfluss der Aus- und Weiterbildung der eigenen Belegschaft führt zu einer höheren Wahrscheinlichkeit, ein bereits im Markt vorhandenes Produkt zu imitieren, eine Prozessinnovation durchzuführen und insbesondere eine nicht-technologische Innovation zu realisieren. Eine kontinuierliche Aus- und Weiterbildung der Belegschaft ermöglicht eine ständige Erweiterung der Wissensbasis im Unternehmen. Dieses Wissen kann dann die Grundlage vor allem von Prozessund nicht-technologischen Innovationen bilden.

Die Digitalisierung der Produktion führt zu einer umfassenden Veränderung von Produktionsprozessen (Porter und Heppelmann 2014). Dies zeigt sich auch in den Ergebnissen der multivariaten Analyse. Unternehmen, die Maßnahmen zur Digitalisierung durchführen, setzen im Zuge dessen insbesondere Prozessinnovation um. Darüber hinaus begünstigen Digitalisierungsmaßnahmen die Innovationsneigung im Allgemeinen und speziell die Umsetzung von nicht-technologischen Innovationen. Im Zuge der Digitalisierung entsteht eine große Menge auswertbarer Daten, die die Unternehmen analysieren können. Das hierdurch generierte Wissen kann sich positiv auf die Innovationstätigkeit der Unternehmen auswirken. Prozessinnovationen erfordern in der Regel Investitionen in neue Anlagen oder Technologien. Folglich haben Unternehmen, die Investitionen durchführen, eine höhere Wahrscheinlichkeit, im Wege der Diffusion Innovationen anzustoßen. Außerdem gehen Investitionsaktivitäten mit einer höheren Wahrscheinlichkeit für Produktimitationen einher.

Zwei weitere Möglichkeiten, externes Wissen für die Generierung eigener Innovationen zu nutzen, sind die Kooperation mit externen Partnern sowie die Inanspruchnahme 
Tabelle 3 Multivariate Ergebnisse: Vergleich alternativer Maßnahmen mit FuE für mittelständische Unternehmen

\begin{tabular}{|c|c|c|c|c|c|c|}
\hline & (1) & (2) & (3) & (4) & (5) & (6) \\
\hline Variablen & Innovation & Markt-neuheit & $\begin{array}{l}\text { Unternehmens- } \\
\text { neuheit }\end{array}$ & Weiterent-wicklung & Prozess-innovation & Nicht-technologisch \\
\hline FuE & $0.203 * * *(0.071)$ & $0.257 * * *(0.045)$ & $0.231 * * *(0.063)$ & $0.218 * * *(0.067)$ & $0.075(0.062)$ & $0.057(0.047)$ \\
\hline Ausbildung & $0.086 *(0.047)$ & $0.065(0.051)$ & $0.019(0.063)$ & $0.138 * *(0.058)$ & $0.158 * * *(0.061)$ & $0.199 * * *(0.059)$ \\
\hline Investitionen & $0.042(0.041)$ & $0.017(0.040)$ & $0.151 * * *(0.052)$ & $0.031(0.051)$ & $0.183 * * *(0.049)$ & $0.018(0.039)$ \\
\hline Digitalisierung & $0.089 *(0.046)$ & $0.001(0.042)$ & $0.047(0.056)$ & $0.071(0.054)$ & $0.180 * * *(0.050)$ & $0.051(0.039)$ \\
\hline Kooperationen & $0.061(0.050)$ & $0.077 *(0.043)$ & $0.041(0.059)$ & $0.137 * *(0.058)$ & $0.085(0.052)$ & $-0.026(0.040)$ \\
\hline Beratung & $0.005(0.049)$ & $0.023(0.043)$ & $0.034(0.059)$ & $-0.001(0.057)$ & $-0.021(0.056)$ & $0.074 * *(0.037)$ \\
\hline Nur Qualität & $-0.007(0.046)$ & $0.052(0.047)$ & $-0.039(0.059)$ & $0.065(0.057)$ & $-0.006(0.057)$ & $0.007(0.042)$ \\
\hline $\log ($ Mitarbeiter $)$ & $0.053 * * *(0.017)$ & $0.016(0.014)$ & $0.037 * *(0.018)$ & $0.040 * *(0.018)$ & $0.010(0.017)$ & $0.023 *(0.012)$ \\
\hline $\log$ (Alter) & $-0.026(0.027)$ & $-0.018(0.027)$ & $-0.015(0.035)$ & $-0.044(0.033)$ & $-0.027(0.034)$ & $0.035(0.024)$ \\
\hline Export: Ja & $0.080 *(0.041)$ & $0.002(0.043)$ & $0.045(0.056)$ & $0.078(0.052)$ & $0.028(0.052)$ & $0.046(0.040)$ \\
\hline West & $0.097 *(0.052)$ & $0.029(0.056)$ & $0.126^{*}(0.070)$ & $0.087(0.068)$ & $0.051(0.065)$ & $-0.011(0.053)$ \\
\hline Distribution & $-0.001(0.052)$ & $0.162 * * *(0.059)$ & $0.112(0.072)$ & $-0.001(0.068)$ & $-0.204 * * *(0.063)$ & $0.132 * *(0.056)$ \\
\hline $\begin{array}{l}\text { unt. Dienstleistun- } \\
\text { gen }\end{array}$ & $-0.013(0.050)$ & $0.111 * *(0.046)$ & $0.024(0.063)$ & $-0.004(0.062)$ & $0.010(0.064)$ & $0.066(0.040)$ \\
\hline $\begin{array}{l}\text { sonst. Dienstleis- } \\
\text { tungen }\end{array}$ & $-0.126(0.082)$ & $0.037(0.062)$ & $0.063(0.096)$ & $-0.072(0.091)$ & $-0.200 * *(0.080)$ & $0.107(0.067)$ \\
\hline Pseudo R-Quadrat & 0.18 & 0.17 & 0.11 & 0.13 & 0.15 & 0.16 \\
\hline Beobachtungen & 331 & 331 & 331 & 331 & 331 & 331 \\
\hline
\end{tabular}

Quelle: Eigene Berechnungen, Standardfehler in Klammern, Signifikanzniveaus: *** $\mathrm{p}<0.01, * * \mathrm{p}<0.05, * \mathrm{p}<0.1$

externer Beratung. Das Eingehen von Kooperationen mit Partnern aus Wirtschaft oder Wissenschaft erweist sich als hilfreich sowohl für die Generierung von Marktneuheiten als auch für die Imitation bereits am Markt vorhandener Produkte. Die Beratung durch Externe begünstigt hingegen die Implementation nicht-technologischer Innovationen.

Bezüglich der Kontrollvariablen ergeben die Regressionsergebnisse folgendes. Große Unternehmen habe eine höhere Wahrscheinlichkeit innovativ zu sein. Gleiches gilt für exportierende Unternehmen im Vergleich zu Unternehmen ohne Exportaktivitäten. Dies gilt vor allem für Produktverbesserungen und nichttechnische Innovationen. Unternehmen, welche ihren Sitz in Westdeutschland haben, weisen eine leicht höhere Innovationstätigkeit auf. Nur wenige Effekte ergeben unsere Schätzungen bezüglich der Branchenzugehörigkeit. Unternehmen in der Branche Distribution setzen eher Marktneuheiten, aber jedoch weniger Prozessinnovationen um als Unternehmen aus dem Produzierenden Gewerbe. Auch Unternehmen, welche im Bereich der unternehmensnahen Dienstleistungen aktiv sind, setzen eher Marktneuheiten um als Unternehmen aus dem Produzierenden Gewerbe. Unternehmen aus den sonstigen Dienstleistungen haben eine deutlich geringere Wahrscheinlichkeit, Prozessinnovationen um zu setzen.

Zur Abschätzung der Bedeutung von FuE im Vergleich zur Relevanz alternativer strategischer Maßnahmen für den Innovationserfolg wurde jedes einzelne Regressionsmodell der jeweiligen Innovationsart nicht auf den nicht-forschenden Mittelstand eingeschränkt, sondern für die gesamten mittelständischen Unternehmen geschätzt. Zusätzlich zu den im obigen Modell enthaltenen Variablen wird eine Dummyvariable für eigene FuE mit in das Modell aufgenommen (Tabelle 3).

Die Schätzungen zeigen, dass eigene FuE für die Realisierung von Innovationen allgemein, im Speziellen jedoch für den Innovationserfolg aller Arten von Produktinnovationen (Neu aus Sicht des Marktes, Imitationen und Produktweiterentwicklungen) eine wesentliche Rolle spielen. Die entsprechenden marginalen Effekte der Variable FuE sind alle signifikant und höher als die Effekte der alternativen strategischen Maßnahmen. Nur in den Modellen für Prozessinnovationen und nicht-technologische Innovationen ist der marginale Effekt für FuE jeweils nicht signifikant. Für diese Innovationsarten ist die eigene FuE im Mittelstand folglich nicht entscheidend. Im Modell für Prozessinnovation werden hingegen für Aus- und Weiterbildung sowie Investitionen und Digitalisierung signifikante Effekte ermittelt, welche auch ökonomisch bedeutsam sind. Gleiches gilt bei nicht-technologischen Innovationen für Aus- und Weiterbildung sowie das Wahrnehmen externer Beratung. Es zeigt sich, dass für das Hervorbringen von Produktinnovationen die eigene FuE-Tätigkeit nur schwer zu ersetzen ist. Prozess- und nicht-technologische Innovationen sind hingegen durch alternative Maßnahmen auch ohne eigene FuE erfolgreich umsetzbar. 


\section{Fazit und Ausblick}

Die innovationspolitische Diskussion auf Unternehmen und Branchen mit hoher FuE-Intensität zu fokussieren - und insbesondere diese als treibende Kraft der deutschen Volkswirtschaft zu interpretieren - ist nicht unproblematisch. Zum einen sind solche FuE-affinen Unternehmen nur für einen relativ geringen Anteil der gesamten Wertschöpfung verantwortlich (Hirsch-Kreinsen 2004). Zum anderen werden über diesen Ansatz FuE-Aktivitäten mit der Innovationsfähigkeit eines Unternehmens gleichgesetzt, ohne dass andere Innovationspfade oder alternative Wettbewerbsstrategien berücksichtigt werden (Kirner et al. 2007). Gegenstand der vorliegenden Studie war es, einerseits zu veranschaulichen, dass FuE nicht den einzigen, zielführenden Weg zu Innovationen darstellen. Andererseits wurden alternative Innovationspfade und Wettbewerbsstrategien für mittelständische Unternehmen beleuchtet.

Auf Basis der vorliegenden Ergebnisse können wir konstatieren, dass FuE keine notwendige Bedingung für Innovationen sind: Ein wesentlicher Teil des Mittelstands innoviert ohne den Einsatz eigener, formaler FuE-Aktivitäten. Dies gilt insbesondere für kleinste, kleine und mittlere Unternehmen. Die Mehrheit der mittelständischen Wirtschaft wählt also Innovationsstrategien abseits von FuE. Die öffentliche Wahrnehmung des Innovationsgeschehens durch den Filter „Forschung und Entwicklung “ und eine hieraus abgeleitete Innovationspolitik klammert einen Großteil innovierender Unternehmen - insbesondere im mittelständischen Unternehmenssegment - aus.

Mittelständische Unternehmen ohne FuE setzen vor allem auf inkrementelle Innovationen sowie Prozessund nicht-technologische Innovationen: So kommt bei nicht-forschenden, mittelständischen Innovatoren insbesondere der Fachkompetenz der Mitarbeiter und der Ausund Weiterbildung eine entscheidende Rolle zu, um die Hervorbringung von Innovationen zu ermöglichen. Soll die Innovationskraft des nicht-forschenden Mittelstands erhalten bzw. gesteigert werden, muss ein besonderes Augenmerk auf die Ressource Humankapital gelegt und die Ausbildungskompetenz des Mittelstands sowie die Verfügbarkeit qualifizierter Fachkräfte gesichert werden.

Die vorliegende Studie liefert erste Einblicke in die Innovationstätigkeit nichtforschender mittelständischer Unternehmen. Neben den Innovationsarten, die von nichtforschenden mittelständischen Unternehmen hervorgebracht werden, wurden außerdem alternative Innovationspfade jenseits der eigene FuE aufgezeigt. Diesbezüglich besteht jedoch noch weiterer Forschungsbedarf. Zukünftige Forschung könnte sich der Frage widmen, welche Rolle unternehmensexterne Quellen wie beispielsweise Kunden oder Zulieferer im Innovationsprozess nicht-forschender mittelständischer Unternehmen spielen. Darüber hinaus sollte die Einbindung mittelständischer Unternehmen in globalen Wertschöpfungsketten und die damit verbundene Innovationstätigkeit analysiert werden. Auch die Möglichkeiten Innovationen im Zuge der Digitalisierung, zum Beispiel durch datenbasierte Geschäftsmodelle, hervorzubringen bedürfen einer näheren Betrachtung. Darüber hinaus sind die Ergebnisse als Korrelationen zu verstehen und ermöglichen keine kausale Interpretation. Zukünftige Forschung sollte daher, so die Datenlage es zulässt, unter Anwendung von Paneldatenmethoden das Innovationsgeschehen in den mittelständischen Unternehmen untersuchen.

Open Access Dieser Artikel wird unter der Creative Commons Namensnennung 4.0 International Lizenz veröffentlicht, welche die Nutzung, Vervielfältigung, Bearbeitung, Verbreitung und Wiedergabe in jeglichem Medium und Format erlaubt, sofern Sie den/die ursprünglichen Autor(en) und die Quelle ordnungsgemäß nennen, einen Link zur Creative Commons Lizenz beifügen und angeben, ob Änderungen vorgenommen wurden. Die in diesem Artikel enthaltenen Bilder und sonstiges Drittmaterial unterliegen ebenfalls der genannten Creative Commons Lizenz, sofern sich aus der Abbildungslegende nichts anderes ergibt. Sofern das betreffende Material nicht unter der genannten Creative Commons Lizenz steht und die betreffende Handlung nicht nach gesetzlichen Vorschriften erlaubt ist, ist für die oben aufgeführten Weiterverwendungen des Materials die Einwilligung des jeweiligen Rechteinhabers einzuholen. Weitere Details zur Lizenz entnehmen Sie bitte der Lizenzinformation auf http://creativecommons. org/licenses/by/4.0/deed.de.

\section{Literatur}

Bender G, Laestadius S (2005) Non-science based innovativeness. On capabilities relevant to generate profitable novelty. Perspect Econ Polit Soc Integr 11(1-2):123-170

Brink S, Kriwoluzky S, Bijedic T, Ettl K, Welter F (2014) Gender. Innovation und Unternehmensentwicklung, Bonn

Cohen WM, Klepper S (1996) Firm size and the nature of innovation within industries: The case of process and product R\&D. Rev Econ Stat 78(2):232-243

Czarnitzki D, Kraft K (2006) R\&D and firm performance in a transition economy. Discussion Paper No. 06-033, Zentrum für Europäische Wirtschaftsforschung (ZEW), Mannheim

DIHK (2017) DIHK-Innovationsreport: Innovationsdynamik rückläufig. Ergebnisse einer Befragung der IHK-Organisation bei 1.700 Unternehmen. Deutscher Industrie- und Handelskammertag e. V. (DIHK), Bereich Energie, Umwelt, Industrie, Berlin

EFI (2016) Gutachten zu Forschung, Innovation und technologischer Leistungsfähigkeit Deutschlands 2016. Expertenkommission Forschung und Innovation (EFI), Berlin

Erdmann V, Koppel O, Lotz S, Plünnecke A (2012) Innovationsmonitor 2012 - Die Innovationskraft Deutschlands im internationalen Vergleich. Eine Studie im Auftrag der Initiative Neue Soziale Marktwirtschaft (INSM), Institut der deutschen Wirtschaft (IW) Köln, Köln

Eurostat, OECD (2007) Eurostat-OECD manual on business demography statistics. https://www.oecd.org/sdd/39974460.pdf. Zugegriffen: 03.04.2021 
Faix WG, Mergenthaler J, Ahlers R-J, Auer M (2014) InnovationsQualität: Über den Wert des Neuen, Steinbeis-Edition. SIBEWissenschaftsreihe, Stuttgart

Freeman C (1992) Formal scientific and technical institutions in the national system of innovation. In: Lundvall B-A (ed) National Systems of innovation: towards a theory of innovation and interactive learning. Anthem Press, London, pp S169-187. https://doi. org/10.7135/UPO9781843318903.010

Freeman C, Perez C (1988) Structural Crisis of Adjustment, Business Cycles and Investment Behaviour. In: Dosi G, Freeman C, Nelson R, Silverberg G, Soete L (Hrsg) Technical Change and Economic Theory. Pinter, London, New York, S 38-66

IfM Bonn (2020) Mittelstandsdefinition des IfM Bonn. Institut für Mittelstandsforschung (IfM) Bonn. https://www.ifm-bonn.org/ definitionen/mittelstandsdefinition-des-ifm-bonn. Zugegriffen: 03.02.2021

Handke M (2005) Innovationen im Mittelstand: Low Tech Unternehmen in Zulieferketten - Das Beispiel der Kunstoff verarbeitenden Industrie. Iwsg Working Papers 03-2005, Institut für Wirtschafts- und Sozialgeographie (Iwsg) Johann Wolfgang Goethe-Universität Frankfurt, Frankfurt

Hirsch-Kreinsen H (2004) "Low-Technology": Ein innovationspolitisch vergessener Sektor. Soziologische Arbeitspapiere 2, Technische Universität Dortmund, Wirtschafts- und Sozialwissenschaftliche Fakultät, Fachgebiet Soziologie, Lehrstuhl Wirtschafts- und Industriesoziologie, Dortmund

Kirner E, Kinkel S, Jäger A (2007) Innovationspfade von Low-, Medium- und Hightech-Unternehmen in der deutschen Industrie. In: Abel J, Hirsch-Kreinsen H (eds) Lowtech-Unternehmen am Hightech-Standort. Nomos Verlagsgesellschaft mbH \& Co, KG, Berlin, pp S165-194. https://doi.org/10.5771/9783845267258-9

Legler H, Grenzmann C, Marquardt R (2005) Forschungs-und Entwicklungsaktivitäten der deutschen Wirtschaft im vergangenen Vierteljahrhundert. Studien zum deutschen Innovationssystem Nr. 2-2006, Niedersächsisches Institut für Wirtschaftsforschung e. V., Sifterverband für die Deutsche Wirtschaft, Berlin

Lundvall B-Å, Johnson B (2017) The Learning economy and the economics of hope. Anthem Press, London. https://doi.org/10.26530/ oapen_626406

Maaß F, Führmann B (2012) Innovationstätigkeit im Mittelstand: Messung und Bewertung. Institut für Mittelstandsforschung (IfM) Bonn, Bonn

Maaß F, May-Strobl E (2016) Der Stellenwert nicht-technologischer Neuerungen im Innovationsgeschehen der mittelständischen Wirtschaft. Institut für Mittelstandsforschung (IfM) Bonn, Bonn

OECD (2010) High-growth enterprises: what governments can do to make a difference. OECD Publishing, OECD Studies on SMEs and Entrepreneurship, Paris. https://doi.org/10.1787/9789264048 782-en

OECD/Eurostat (2005) Oslo manual: guidelines for collecting and interpreting innovation data, 3rd edn. OECD/Eurostat, Paris. https://doi.org/10.1787/9789264013100-en

Pavitt K (2003) The Process of Innovation. Working Paper No. 89, Science and Technology Policy Research, The Freeman Centre University of Sussex, Brighton

Porter ME, Heppelmann JE (2014) Wie smarte Produkte den Wettbewerb verändern. Harv Bus Manag 12(2014):34-60

Rammer C, Köhler C, Murmann M, Pesau A, Schwiebacher F, Kinkel S, Kirner E, Schubert T, Som O (2010) Innovationen ohne Forschung und Entwicklung: Eine Untersuchung zu Unternehmen, die ohne eigene FuE-Tätigkeit neue Produkte und Prozesse einführen. Studien zum deutschen Innovationssystem Nr. 15-2011, Zentrum für Europäische Wirtschaftsforschung (ZEW), Fraunhofer-Institut für System- und Innovationsforschung (ISI), Mannheim, Karlsruhe

Rammer C, Berger M, Doherr T, Mud M, Hünermund P, Iferd Y, Peters B, Schubert T (2017) Innovationsverhalten der deutschen Wirtschaft: Indikatorenbericht zur Innovationserhebung 2016. ZEW Innovationserhebung - Mannheimer Innovationspanel (MIP), Zentrum für Europäische Wirtschaftsforschung (ZEW), Mannheim

Rammer C, Aschhoff B, Doherr T, Peters B, Schmidt T (2019) Innovationen in der deutschen Wirtschaft: Indikatorenbericht zur Innovationserhebung 2018. ZEW Innovationserhebung - Mannheimer Innovationspanel (MIP), Zentrum für Europäische Wirtschaftsforschung (ZEW), Mannheim

Rammer C, Behrens V, Doherr T, Hud M, Köhler M, Krieger B, Peters B, Schubert T, Trunschke M, Burg JVD (2020) Innovationen in der deutschen Wirtschaft: Indikatorenbericht zur Innovationserhebung 2019. ZEW Innovationserhebung - Mannheimer Innovationspanel (MIP), Zentrum für Europäische Wirtschaftsforschung (ZEW), Mannheim

Schumpeter JA (1961) Konjunkturzyklen. Vandenhoeck \& Ruprecht, Göttingen

Shefer D, Frenkel A (2005) R\&D, Firm Zize and innovation: an empirical analysis. Technovation 25(1):25-32

Tidd J, Bessant JR, Pavitt K (2018) Managing innovation: integrating technological, market and organizational change, 6th edn. Wiley, Chichester

Trantow S, Hees F, Jeschke S (2011) Die Fähigkeit zur Innovation Einleitung in den Sammelband. In: Jeschke S, Isenhardt I, Hees F, Trantow S (eds) Enabling innovation. Innovative capability-german and international views. Springer, Berlin, pp S1-14. https:// doi.org/10.1007/978-3-642-24503-9 